\title{
BMJ Global Health Length of maternal schooling and children's risk of malaria infection: evidence from a natural experiment in Uganda
}

Kazuya Masuda

To cite: Masuda K. Length of maternal schooling and children's risk of malaria infection: evidence from a natural experiment in Uganda. BMJ Global Health 2020;5:e001729. doi:10.1136/ bmjgh-2019-001729

Handling editor Sanni Yaya

Received 21 May 2019

Revised 10 December 2019

Accepted 22 December 2019
D Check for updates

(c) Author(s) (or their employer(s)) 2020. Re-use permitted under CC BY-NC. No commercial re-use. See rights and permissions. Published by BMJ.

Hitotsubashi University Institute of Economic Research, Kunitachi, Tokyo, Japan

Correspondence to Dr Kazuya Masuda masuda@ier.hit-u.ac.jp

\section{ABSTRACT}

Introduction An estimated 216 million cases of malaria occurred worldwide every year. Cross-sectional studies have reported negative association between maternal education and child malaria risks; however, no randomised trial or quasi-experimental study using a natural experiment has confirmed a causal relationship between these two factors. I used the free primary education reform in Uganda to assess the causal effects of maternal schooling on children's risk of malaria infection.

Methods Malaria biomarkers of children aged $<5$ years were collected from the 2009 and 2014 Uganda Malaria Indicator Surveys $(n=5316)$. In 1997, the government eliminated tuition requirements in primary schools, which increased the educational attainment of the affected cohorts. Using exposure to the reform as an instrumental variable, I used a two-stage least squares approach to estimate the causal effects of maternal year of education on the probability that a child would contract malaria at the time of the survey. I also evaluated the cost-effectiveness of primary schooling as a malaria control intervention. Results One extra year of maternal education reduced children's risk of malaria infection by 7.5 percentage points $(p=0.057)$ from baseline $(34.9 \%)$. The length of maternal education was also positively associated with insecticidetreated bednet usage by their children. The results were robust to a variety of sensitivity tests. Primary schooling for women was a cost-effective intervention to reduce children's malaria infection.

Conclusion Improving access to primary education could be a cost-effective measure to reduce malaria prevalence among children of educated mothers aged $<5$ years in malaria-endemic countries.

\section{INTRODUCTION}

Malaria remains a major global health issue worldwide, with an estimated 216 million cases in 2016. ${ }^{1}$ While existing cross-sectional studies suggest that formal education for mothers could reduce children's risk of a malaria infection, little is known about the causal relationship between these two factors. ${ }^{2-4}$

Mothers' education may reduce the incidence of malaria infection among their

\section{Key questions}

What is already known?

- Cross-sectional studies have reported a negative association between maternal education and child malaria risks.

- However, this association does not represent 'causality', and no study has taken advantage of an experimental design or exogenous variation in maternal education created by a natural experiment.

What are the new findings?

- The present study is the first to estimate the causal effects of length of maternal education on malaria incidence in children by using exposure to educational reform as a natural experiment.

- Primary schooling for women had large protective effects on malaria infection in children aged younger than 5 years.

- The length of maternal education was also positively associated with insecticide-treated bednet usage by their children.

What do the new findings imply?

- Improving access to primary education for women could be a cost-effective strategy to combat malaria in malaria-endemic countries, in addition to its other private and societal benefits.

- My findings support the hypothesis that improved malaria vector control by mothers is a probable mechanism for increased bednet usage, and therefore, they help researchers to understand how longer length of female education increases the bednet usage in the overall population.

- Further research is required to test whether and to what extent maternal education interventions together with insecticide-treated net distribution would reduce the incidence of malaria among children.

children for several reasons: 1) improved ability to gather and process information on the transmission mechanism and vector control strategies, ${ }^{4-11}$ 2) different social norms associated with different social networks, 3) tendency to migrate to an area 
with less malaria infection risks, 4) access to more financial resources through one's own labour force participation, ${ }^{9} 105$ ) having a partner with a high socioeconomic status $^{9}{ }^{10}$ and 6) having more decision-making power within one's marriage and household. ${ }^{9}{ }^{10}$ These changes may result in better use of vector control strategies, including insecticide-treated bednets (ITNs), ${ }^{612} 13$ indoor residual spraying (IRS) and antimalaria drug treatments. ${ }^{46714}$ In addition to the implications concerning controlling childhood malaria, this study contributes to the debate on the causal effects of maternal education on the health of children in general. ${ }^{15-17}$

Identifying the causal effects of maternal education on malaria incidence among their children is empirically not straightforward because mothers' educational attainment is likely to be correlated with various confounding factors, (ie, household socioeconomic status, risk preference and innate ability), which can be difficult to control for in observational studies. For this reason, bivariate or multivariate regression analyses may produce a spurious association between maternal education and childhood malaria, and causal relationships cannot be inferred. ${ }^{2}$ To my knowledge, no randomised trial or quasi-experimental study using a natural experiment has ever confirmed whether, or to what extent, length of maternal formal education reduces the incidence of malaria infection among their offspring.

To estimate the causal effects of maternal education on childhood malaria control, this study exploited acrosscohort variation in women's educational attainment generated by exposure to the 1997 free primary education reform in Uganda. In January 1997, the government abolished tuition and parent-teacher association fees in public primary schools. Consequently, the educational attainment of the affected cohort increased. ${ }^{18}$ Specific cohorts, born in 1982 or later, have been disproportionately affected, and their children's risk of malaria infection has been affected only through the change in the length of maternal formal education. Thus, this reformas a natural experiment-provides an ideal setting to study the effects of maternal education on childhood malaria control. To uncover the potential channel through which maternal education affects children's risk of malaria infection, I estimated its effects on ownership and use of ITNs. I also evaluated the cost-effectiveness of primary schooling as a malaria control intervention.

Uganda's formal education consists of primary (7 years), lower secondary (4 years) and upper secondary education ( 2 years). The national law stipulates that children start 7 years of primary education at age 6 , but before the reform started in 1997, delayed school entry and grade repetition were not uncommon. The primary level gross enrolment rate was $70 \%$ in $1996{ }^{19}$ Public primary schools previously relied financially on private resources. Parents contributed to the majority of school inputs such as tuition, parent-teacher association fees and uniform costs. These costs per child at public primary schools amounted to $62 \%$ of the average annual household expenditure. This financial burden made it difficult for many, particularly poor, parents to afford school costs. $^{18}$

In January 1997, the tuition and parent-teacher association fees in public primary schools were removed. ${ }^{20} 21$ This reform effectively increased the primary-level gross enrolment ratio, and this increase was particularly large among girls. ${ }^{10} 182223$ Primary attendance rates consistently rose from $59.7 \%$ in 1992 to $83.2 \%$ in 1999 for girls, and it also improved from $64.3 \%$ in 1992 to $84.0 \%$ in 1999 for boys. By dividing the sample by quintile of the per capita expenditure distribution, among the poor household (first quintile), it increased from $45.7 \%$ to $75.1 \%$, whereas among the wealthy household (fifth quintile), it slightly increased from $81.7 \%$ to $89.0 \%$ during the same period. ${ }^{18}$

\section{METHODS}

\section{Study population and data source}

Uganda is one of the most severe malaria-endemic countries: the malaria incidence rate was 272 per 1000 people in 2016. ${ }^{24}$ Plasmodium falciparum is the predominant Plasmodium species found in Uganda. ${ }^{25} 26$

The data used in this study came from the 2009 and 2014 Uganda Malaria Indicator Surveys (UMIS), which were nationally representative, repeated, cross-sectional, household surveys that included malaria biomarker collection for children younger than 5 years of age. ${ }^{27} 28$ In the 2009 and 2014 surveys, 4760 and 5802 households were interviewed, and the response rates were $96 \%$ and $97 \%$, respectively. All girls/women aged 15-59 years were eligible for the individual interview. In addition, I obtained data from the UMIS concerning testing for malaria in children aged 0-59 months using blood obtained by finger-prick. Blood slides were read in the laboratory for the presence of Plasmodium parasites and to determine the parasite species. Verbal, informed consent for each test was granted from the children's parents. All children aged 0-59 months in the households selected for UMIS were eligible for biomarker collection, and $97 \%$ of eligible children were tested for malaria with microscopy in both years. ${ }^{25}{ }^{26}$ Key information on birth year, age and mothers' educational attainment were available for $99 \%$ of the respondents. Maternal age in the study population ranged from 18 to 41 years, with a mean of 28.1 (SD 5.0) in the full sample. Mean child age was 28.9 months. Mean length of maternal schooling was 4.8 years (SD 3.6) in the 2009 UMIS and 4.9 years (SD 4.1) in the 2014 UMIS. Before the exploratory analysis, I decided to excluded children of mothers born in 1973 or earlier and those born in 1992 or later because those born in 1992 or later were aged 17 years or younger in the 2009 UMIS, and with symmetric bandwidth, those women born in 1973 or earlier should be excluded and were likely to be exposed to other education reform. ${ }^{10}$ Other predetermined exclusion criteria were as follows: 1) children of mothers who were aged $<18$ years were excluded because 
they may still have been receiving formal education at the time of the survey; 2) I also excluded women with no children aged $<5$ years because my primary interests were the effects of maternal education on childhood malaria control.

\section{Exposure and outcome variables}

Primary exposure was defined as mothers' educational attainment (in years) at the time of the survey. Mothers' educational attainment was calculated based on the final grade completed. The primary outcome was their children's malaria status at the time of the survey. I defined malaria status based on the results of the microscopy testing to determine the presence of Plasmodium infection. I define binary variable which takes value of unity if the respondent woman born in a cohort exposed to 1997 reform, otherwise zero. Considering the share of individuals attending primary school in 1996 by age, those born in 1982 or later (ie, $\leq 14$ years as of 1 January 1996) were defined as 'exposed'. ${ }^{10}$ In the analysis, I controlled for maternal year of birth, maternal ethnicity (dummy variable indicating the major ethnic group, which includes baganda, banyankore, iteso, basoga and is otherwise 0 ), children's age in months and year of the survey. Mothers' ethnicity and children's age in months were measured based on the mothers' self-reported information. Survey year was recorded by the field workers.

\section{Statistical analyses}

To study to what extent the estimated association is biassed if confounding factors are not controlled for, I first evaluated the association between maternal education and children's malaria status using a multivariate ordinary least squares (OLS) regression analysis; next, to reduce the omitted variable bias, I have presented results from the two-stage least squares (2SLS) instrumental variable (IV) models. ${ }^{27}$ This study reports the analysis in three steps. First, I tested whether women from the exposed cohorts disproportionately attained more education compared with those who were not exposed (first stage). I estimated the effects of the reform on mothers' educational attainment using an OLS regression model (linear regression model). To provide an intuitive explanation of the identification strategy, I have also graphically presented whether the reform increased the probability that women completed grades $1-7$ of primary education or greater. Second, to describe how much the incidence of malaria infection differs between the children of the treatment group and those of the control group, I evaluated intention-to-treat (ITT; or reduced form) effects of the reform on children's malaria status. A quasi-experimental design (natural experiment), under the assumption that cohorts near the exposure cut-off are plausibly similar, has allowed me to study whether the probability that a child contracts malaria at the time of the survey declined among the exposed cohort compared with the unexposed cohort. Third, I have presented the results from the 2SLS estimates using dichotomous variables for the 1997 reform exposure as an (IV) to show how 'an additional year of maternal education' affects children's malaria risks. Under several assumptions, the estimated effect size was interpreted as local average treatment effects (LATE) and described as the ratio of the ITT effects to the difference in the maternal educational attainment caused by the reform (IV estimate=ITT estimate/first stage estimate). LATE describes the average treatment effects on a subset of the population-so-called compliers-who attend school longer if they are exposed to the reform but do not attend in the absence of the reform. ${ }^{28}$

To isolate the treatment effects of maternal education on children's risk of malaria infection from the confounding factors, I first controlled for the full set of children's age-in-months indicators to explain non-linear relationships between children's age and malaria risk in all three above-mentioned analyses. Second, I included the indicators for mothers' ethnicity to account for biological diversity in the multivariate regression analyses. Third, I included the indicators for survey year. Lastly, the regression model included mothers' year of birth in a linear form to adjust for gradual and continuous trends in maternal educational attainment (in the first-stage model), and in children's risk of malaria infection (in the ITT and IV models). Reform exposure effects are described as the change in the intercept for the cohort exposed to the reform (ie, born $\geq 1982$ ) compared with those who were not exposed (ie, born $\leq 1981$ ). When I explored the behavioural mechanism through which maternal education reduces children's risk of malaria infection, the outcome variable was replaced by a binary indicator for using ITNs last night and sleeping under a bednet last night. A separate model was estimated for each outcome.

To interpret the estimated effects of maternal education on children's risk of malaria as causal effects for the compliant population, four identification assumptions must be satisfied (figure 1). First, the IV (Z) must have had a sufficient impact on individual educational attainment $(\mathrm{S})$. This condition is empirically testable, and the results suggests that IV has large, positive and significant effects on maternal formal education. Second, IV (Z) must be unrelated to unobserved confounding factors (U), such as household income, psychological traits and innate ability, conditional on the observed covariates (X). This implies that a cohort born just before 1983 and those born just after 1983 are plausibly similar in the absence of the programme after controlling for a cohort linear trend, survey year and ethnicity. In other words, if, for example, IV is positively correlated with unobserved factors, such as household income, then the estimated effects of the length of maternal education on children's risk of malaria infection captures the effects of household income on children's risk of malaria infection in addition to its true effects. A combination of the two survey rounds allowed me to control for survey year periodic effects on women's educational attainment (in the first-stage 


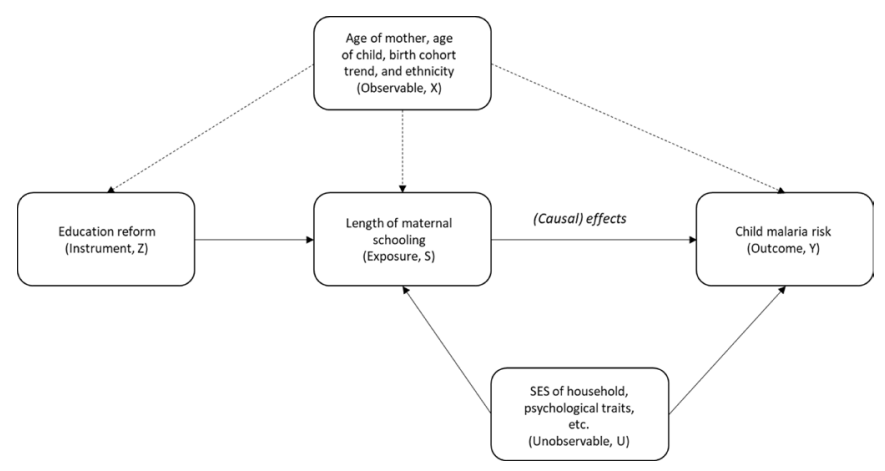

Figure 1 Causal diagram. This diagram explains the identification assumptions under the two-stage least squares method. I assume that, conditional on $X, Z$ is a valid instrument if 1) $Z$ is strongly correlated with $S$ (relevance), 2) $Z$ is not associated with $U$ and 3) $Z$ affects $Y$ only through its effects on $S$ (exclusion restriction). Under an additional assumption, 4) Z changes S only in one direction (monotonicity), the estimated treatment effects are interpreted as local average treatment effects.

model) and children's risk of malaria infection (in the ITT and IV models). The model implicitly controls for maternal age effects by including the full set of the survey indicators and linear birth cohort trend.

To test the robustness of the results to the presence of non-linear cohort trends in maternal education and children's risk of malaria, I also included quadratic terms for maternal year of birth as control variables, allowed the slope of the trend to differ between exposed and unexposed cohorts, and limited the window of the observation to the narrower set of birth cohort. Overall, the main results of the current study are not sensitive to the selection of the model specification or sample. To test the robustness of the results using a different identification strategy, I also exploited the across-district variation in the primary school completion rates among the unexposed women to estimate the treatment effects using the difference-in-differences method. The idea behind this strategy is that the reform should have had larger impacts in the district where more women failed to complete 7 years of primary education in the absence of the reform than in the district where most women completed it in the absence of the reform. ${ }^{9}$

Third, to obtain a consistent estimate of the causal effects of maternal education on children's risk of malaria infection, I assumed that the IV (Z) affects the primary outcome $(\mathrm{Y})$ only through its effects on maternal year of education $(\mathrm{S})$. This so-called exclusion restriction is plausible because the timing and content of the reform were exogenously designed and implemented by the supply side (ie, government), and it should not have resulted in cohort-specific effects other than expanding primary school access. Relatedly, a key condition for my identification strategy was that no other interventions or reform would have affected the children's risk of malaria infection among mothers who were exposed to the 1997 reform. This is because, in the presence of such a reform, the coefficient of our IV (exposure indicator) in the first stage and ITT analysis may capture the effects of the 1997 education reform and the other reform simultaneously, and possibly overestimate the true effect size of the 1997 education reform's effects on children's malaria risks, and consequently the effects of the length of maternal education on children's malaria risks. To test if children's risk of malaria infection changed even in the absence of the 1997 education reform, a placebo test was conducted-I assessed the effects of reform exposure on the children of mothers with no education who were unlikely to be affected by the reform. Fourth, to interpret the estimated treatment effects as LATE for complier, monotonicity assumption must be maintained. Namely, it must be maintained that the reform either increased (complier) or had no effects (nevertaker or always taker) on women's educational attainment, and no one in the exposed cohort reduced the length of schooling because of exposure to the reform (ie, defier).$^{28}$ The presence of a defier is theoretically possible; however, it is practically very unlikely given that the reform vastly reduced the price of receiving primary education. To evaluate the cost-effectiveness of the primary schooling to prevent children's malaria infection, I calculated the cost of providing an extra year of primary education in Uganda to estimate the cost per malaria incidence averted and per disability-adjusted life-year (DALY) averted.

The empirical approach of the current study is theoretically equivalent to a fuzzy regression discontinuity design. ${ }^{29}$ To understand the relationship between length of maternal education and children's malaria risk, the basic regression model is described as follows:

$$
Y_{i t}=\beta_{0}+\beta_{1} S_{i t}+\dot{x}_{i t} \gamma+\epsilon_{i t}
$$

Where $Y_{i t}$ indicates the child's malaria status of mother $i$ born in year $t, S$ is the length of maternal education, and $x^{\prime}$ is the vector of the individual characteristics of the mother. The parameter of our interest is $\beta_{1}$, which shows the marginal effects of maternal education on a child's risk of malaria infection. Estimating equation (1) using OLS would produce a consistent estimate of $\beta_{1}$ if length of maternal education is randomly assigned to each woman.

In reality, however, length of maternal education is endogenously determined, and OLS estimates tend to be biassed. This is because, for example, women from wealthy households tend to attain longer years of schooling, and the higher level of the household's wealth may directly improve the child's risk of malaria infection. It is also possible that women born in an urban community may attend school longer years, and children's risk of malaria is also lower in such communities possibly due to improved hygiene and lower density of mosquitos. In these cases, estimated $\beta_{1}$ by OLS will be overestimated.

In order to overcome such omitted variable bias, I used the exogenous variation in the maternal education created by an across-cohort variation in the exposure to 
the 1997 education reform in Uganda in line with the fuzzy regression discontinuity design.

The first stage model is as follows:

$$
\widehat{\mathrm{S}}_{\mathrm{it}}=\pi_{0}+\pi_{1} \text { Treat }_{\mathrm{t}}+\mathrm{f}(\text { year of birth }-1982)+\eta_{\mathrm{it}}
$$

Where Treat $t_{t}$ is a binary variable that takes the value of unity if the respondent woman was born in 1982 or later (ie, $\leq 14$ years as of 1 January 1996).

I used equation (2) to obtain the predicted value of $S_{i t}$ , and I used this predicted value in the following second stage model:

$$
\mathrm{Y}_{\mathrm{it}}=\delta_{0}+\delta_{1} \widehat{\mathrm{S}}_{\mathrm{it}}+\mathrm{g}(\text { year of birth }-1982)+\mathrm{v}_{\mathrm{it}}
$$

Given that the order of polynomial and the bandwidth are equivalent in (2) and (3), the system of the equation is numerically equivalent to the 2SLS. Before data analysis, the order of the polynomial is determined as first order (linear function) by following De Neve $e t a l^{30}$ In other words, we estimated the discontinuity in the outcome after controlling for age trends. In addition, in the exploratory analysis, the robustness of the results were tested with different forms of polynomials and bandwidth. Our identification assumption is that the characteristics of the women on either side of the cut-off are plausibly similar (or continuous) after controlling for the linear trend by polynomial function. Online supplementary figure S2 tests the validity of this assumption by showing the change in the exogenous characteristics of the mother by birth cohort. The results suggest that the characteristics of the mothers, for example, length of maternal education of the mother of a child, show no sign of a discontinuous jump around the cut-off, supporting the validity of the continuity assumption.

\section{Patient and public involvement}

This research was done without patient involvement. Patients were not invited to comment on the study design and were not consulted to develop patient relevant outcomes or interpret the results. Patients were not invited to contribute to the writing or editing of this document for readability or accuracy.

\section{RESULTS}

Malaria biomarkers for children aged 0-59 months were available for 5316 children (figure 2 and table 1). The unadjusted association between the length of maternal formal education and child malaria infection were almost monotonically negative (figure 3).

This negative association between maternal education and the children's risk of malaria infection remained strong even in the estimates using a naive multivariate linear regression model (online appendix, p. 9). This suggests that an extra year of maternal formal education is associated with a 2.1 percentage point $(\mathrm{SE}=0.2)$ decrease in the probability that her child will contract malaria at the time of the survey. This significant association, however, may be not causal because of the possible association between observed maternal education (S)
Children of mothers interviewed in the 2009 and 2014 UMIS who were eligible for malaria biomarker collection and who had data for mother's birth year, ethnicity, and educational attainment $(n=7147)$

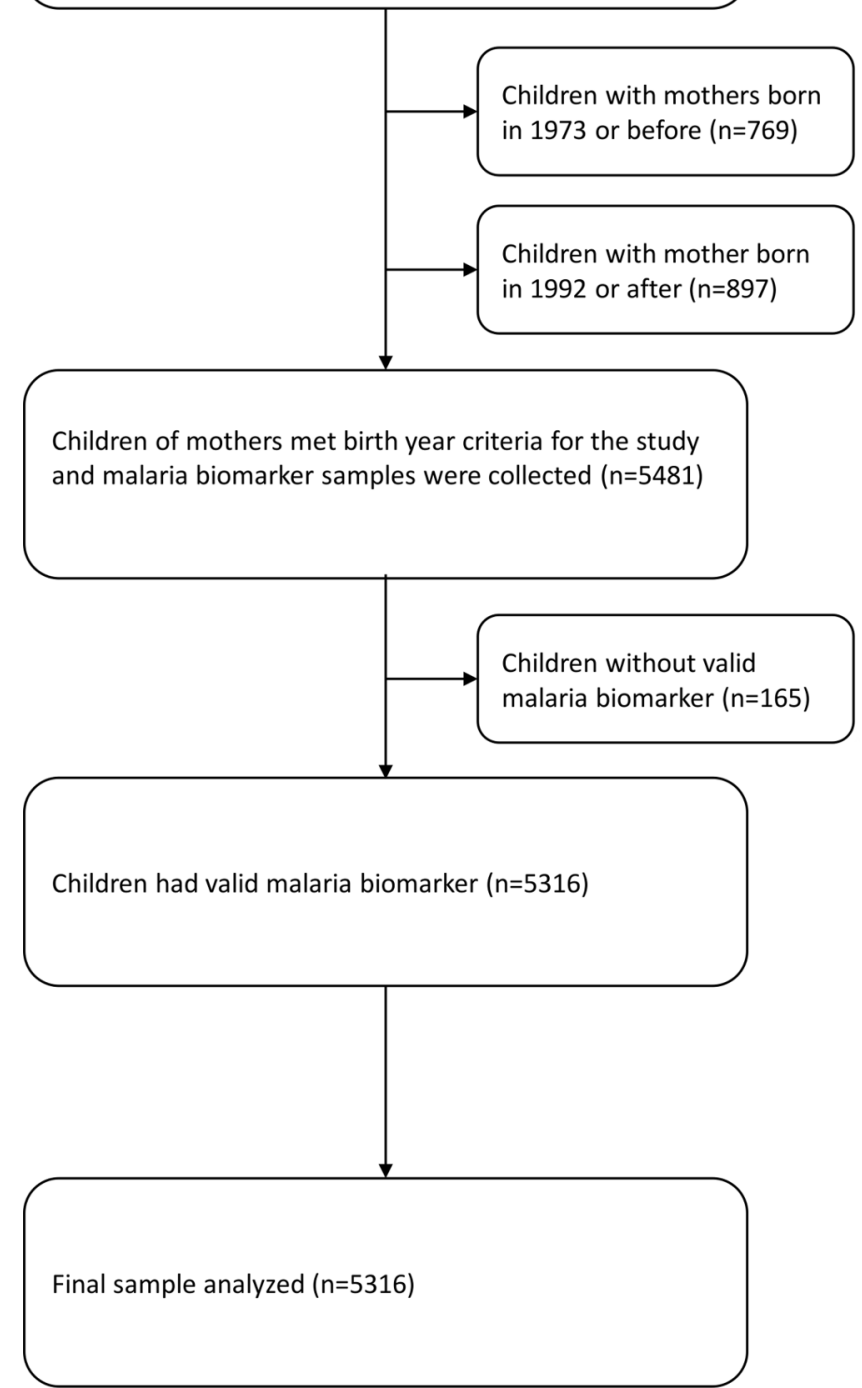

Figure 2 Study participant flow through the Uganda Malaria Indicator Surveys (UMIS) in 2009 and 2014.

and unobserved confounding factors (U). Therefore, I exploited the exogenous variation in the length of maternal education as if it was randomly caused by the 1997 reform to obtain a 2SLS estimate of its causal effects on children's risk of malaria infection.

First stage: the effects of the reform exposure on the mother's educational attainment

The first-stage model shows that the reform increased maternal educational attainment by 0.65 years on average $(\mathrm{SE}=0.20 ; \mathrm{p}<0.0001 ;$ table 2$)$. Figure 4 depicts that share of mothers completing each grade of primary education grew slowly until the birth cohort born in 1981. This share disproportionately increased among the $\geq 1982$ cohort. By contrast, I did not find the increase in the probability 
Table 1 Participants' characteristics

\begin{tabular}{|c|c|c|}
\hline & UMIS (2009) & UMIS (2014) \\
\hline & $(n=2501)$ & $(n=2815)$ \\
\hline Child malaria positive & $1002(40.1 \%)$ & $528(18.8 \%)$ \\
\hline Children's age (months) & 27.5 (16.9) & $30.1(17.0)$ \\
\hline Mothers' age (years) & $26.2(4.6)$ & $29.8(4.8)$ \\
\hline Mothers' year of schooling & $4.8(3.6)$ & $4.8(4.1)$ \\
\hline $\begin{array}{l}\text { Has at least } 7 \text { years of } \\
\text { schooling }\end{array}$ & 759 (30.3\%) & $891(31.7 \%)$ \\
\hline $\begin{array}{l}\text { Child contracts moderate } \\
\text { anaemia }\end{array}$ & $1064(42.6 \%)$ & 799 (28.4\%) \\
\hline $\begin{array}{l}\text { Owned mosquito net last } \\
\text { night }\end{array}$ & 1689 (67.5\%) & 2725 (96.8\%) \\
\hline $\begin{array}{l}\text { All children aged }<5 \text { years } \\
\text { slept under net }\end{array}$ & $922(37.0 \%)$ & $2076(74.0 \%)$ \\
\hline
\end{tabular}

Mothers were aged $\geq 18$ years at the time of the survey, born between 1974 and 1991, and whose children had valid results for microscopy malaria testing. Data shown as $\mathrm{n}(\%)$ or mean (SD). UMIS, Uganda Malaria Indicator Surveys.

that women completed the eighth grade (first grade of secondary education) or more among the $\geq 1982$ cohort.

Likewise, the upper panel of figure 5 demonstrates the effects of the reform exposure to maternal educational attainment in the UMIS with the normalised age of the women in 1997 presented along the horizontal axis. The vertical line indicates the 1982 cohort that I consider to be the treatment group. The women on the left-hand side of the vertical axis are considered to be the control group, whereas those on the right-hand side are considered the treatment group. The increase in the length of maternal education and the probability of completing 7 years of primary education for the 1982 cohort is evident, and I use this discontinuity as a natural experiment to see whether children's malaria risk falls at this same timing in the fuzzy regression discontinuity design approach.

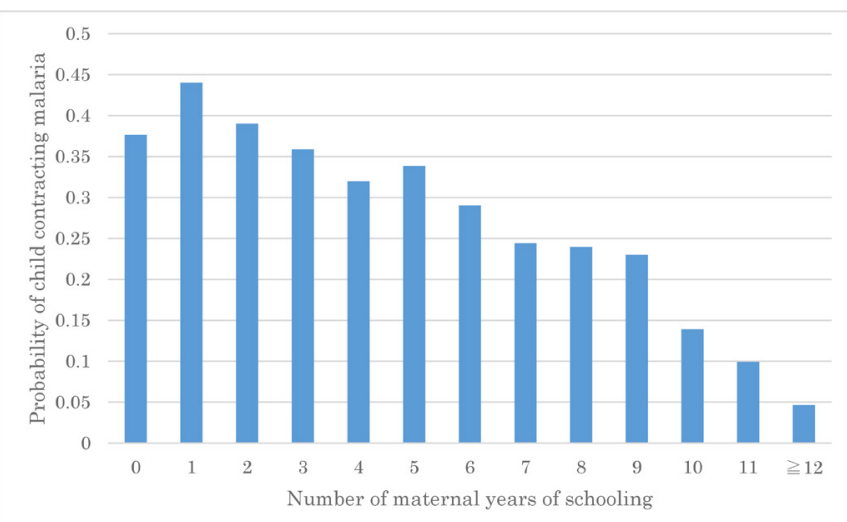

Figure 3 Probability that children contracted malaria at the time of the survey by maternal education. The sample includes children aged 0-59 months at the time of the survey and their mothers born between 1974 and 1991, and who had valid results for microscopy malaria testing.
The effects of the reform exposure on the child's malaria and anaemia status

The lower panel of figure 5 presents the change in the children's risk of malaria infection and anaemia by birth cohort of the mothers. I consistently found a discontinuous decline in the probability that a child would contract malaria and anaemia at the same time when maternal education increased substantially.

The second row of table 2 shows the ITT effects of the reform on children's risk of malaria infection. The reform reduced the probability that children of exposed mothers will contract malaria at the time of the survey by 7.5 percentage points, on average $(\mathrm{p}=0.057)$.

\section{Second stage: the effects of the length of the maternal education on the child's malaria and anaemia status}

To uncover whether the length of maternal education causally reduces the child's risk of malaria infection, I have presented the results from the 2SLS-IV model. In the 2SLS model (row 3 of table 2), the probability that a child contracts malaria decreased by 4.8 percentage points as mothers attained one extra year of education on average $(p=0.042)$. Compared with the probability that children of mothers born in 1980 contract malaria $(35.3 \%)$, this effect size is as large as a $21 \%$ reduction per additional year of maternal schooling.

I conducted multiple sensitivity analyses for the 2SLS estimates (table 3 ) and detailed discussions are presented in the online supplementary appendix. Overall, the results were robust to different observation windows, and functional form of the birth cohort trend in exposure and outcome. The difference-in-differences estimates were qualitatively similar, although different IV theoretically provides different LATE because compliers are different. The placebo test indicates that the reform had negligible effects on the women with no schooling who were unlikely to be exposed to the reform.

Coefficient and SEs are multiplied by 100 and reported on a percentage point scale. Models 1-6 were estimated by IV-2SLS method, in which binary indicator for being born 1982 or later was used as an excluded IV for maternal years of schooling. Model 7 was estimated by IV-2SLS method, in which binary indicator for being born 1979 or later was used as an excluded IV for maternal years of schooling. Model 8 is estimated by OLS method. The entire model controls for a full set of children's age-inmonths indicators, linear term of maternal year of birth, indicator for survey year, indicator for survey month, indicator for child sex, indicator for ethnic groups and maternal age $(* \mathrm{p}<0.05, \quad * * \mathrm{p}<0.01, \quad * * * \mathrm{p}<0.001)$. No weights were used.

UNESCO estimated the cost per primary student per year as US\$34.2 in Uganda between 2010 and 2013, on average. ${ }^{31}$ Since an additional year of maternal education reduced the probability that her child contracts malaria at the time of the survey by 7.5 percentage points, the cost per malaria infection among children aged $<5$ years averted amounts to US $\$ 456$. Referring to 
Table 2 Regression results

\section{Exposure}

Schooling ( $n=5316 ; 4.4$ years of education pre-reform)

Model 1: first stage

Reform indicator

Malaria ( $n=5316 ; 34.9 \%$ malaria positive pre-reform)

\begin{tabular}{|c|c|c|c|}
\hline Model 2: intention-to-treat & Reform indicator & Malaria positive & $-4.8^{\star \star}(2.4)$ \\
\hline Model 3: 2SLS (IV) & Years of schooling & Malaria positive & $-7.5^{\star}(3.9)$ \\
\hline \multicolumn{4}{|c|}{ Anaemia $(n=5316 ; 41.2 \%$ moderate or serious anaemia prereform) } \\
\hline Model 3: 2SLS (IV) & Years of schooling & Anaemia & $-8.2^{\star}(4.3)$ \\
\hline \multicolumn{4}{|c|}{$\begin{array}{l}\text { Vector control ( } n=5316 ; 77.5 \% \text { owns net pre-reform; } n=4558 ; 47.9 \% \text { all children aged }<5 \text { years slept } \\
\text { under net pre-reform) }\end{array}$} \\
\hline Model 4: 2SLS (IV) & Years of schooling & $\begin{array}{l}\text { All children aged }<5 \text { years slept } \\
\text { under a bednet last night }\end{array}$ & $10.1^{\star *}(4.8)$ \\
\hline
\end{tabular}

For malaria, anaemia and vector control outcome, regression coefficient and SEs were multiplied by 100 and reported on a percentage point scale. Per cent malaria positive refer to the prevalence among children of mothers in the 1980 cohort. Model 1 was estimated by OLS method; model 2 by OLS linear probability method; model 3 by IV-2SLS method, in which binary indicator for being born 1982 or later was used as an excluded IV for maternal years of schooling and model 4 by IV-2SLS with sample owns mosquito net. All model controls for full set of children's age-in-months indicators, a linear term of maternal year of birth, indicator for survey year, indicator for survey month, indicator for child sex, indicator for ethnic groups and age of the mother. No weights were used. Kleibergen-Paap Wald rk F statistic in the first stage models were 10.4 .

${ }^{\star} \mathrm{p}<0.10,{ }^{\star \star} \mathrm{p}<0.05,{ }^{\star \star *} \mathrm{p}<0.01$.

IV, instrumental variable; OLS, ordinary least square; 2SLS, two-stage least square.

the WHO standard, the intervention costs less than the GDP per capita per DALY averted are considered a very cost-effective intervention. Given the calculation shown in the online supplementary appendix, a malaria infection in children aged 5 years in Uganda led to 0.5 DALY lost. Given the total fertility rate of 5.6 in Uganda, ${ }^{32}$ the resulting cost-effective ratio per DALY averted of maternal primary schooling is US\$162.9. According to the World Bank, GDP per capita in Uganda in 2017 was US $\$ 604,{ }^{32}$ suggesting that promoting primary education is a very cost-effective intervention to prevent child malaria morbidity. Compared with existing interventions, primary schooling for mothers is more expensive than

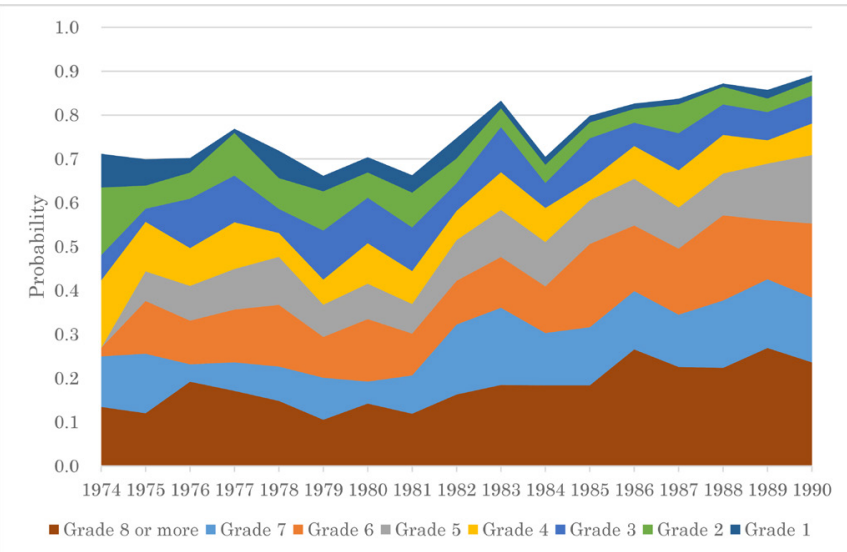

Figure 4 Maternal educational attainment by birth cohort. Shows the probability that a mother completed certain grade of education by the time of the survey. is chemoprophylaxis; long-lasting ITNs; intermittent preventive-treatment in infants and antimalarial drugs, including parenteral artenusate, dihydroartemisinin piperaquine and artemether lumefantrine; however, it is comparable to IRS and RTS, S/AS01 vaccine. ${ }^{33-36}$

\section{Mechanism}

A natural next question is how maternal education reduced children's risk of malaria. In the 2SLS model, the probability that a child used a mosquito net in the night before the interview increased by 10.1 percentage point as the length of the mother's education rose 1 year. Mosquito net ownership, however, was not associated with maternal education. Figure 6 assesses the effects of maternal education on various mechanism outcomes, showing that knowledge improvement is likely to help mothers place a better investment in the health of their children as well.

\section{Generalisability: replication in Malawi}

To test the external validity, I used the 1994 free primary education reform in Malawi for replication, and the results are qualitatively similar (row 7 in table 3 and online supplementary appendix table S4). Malawi is another country where the malaria endemic is most severe. The malaria incidence rate was 188.8 per 1000 people in $2015 .^{37}$ P. falciparum is the predominant Plasmodium species found in Malawi. ${ }^{38-40}$

The data used in this replication came from the 2012, 2014 and 2017 Malawi Malaria Indicator Surveys (MMIS), which were nationally representative, repeated, 

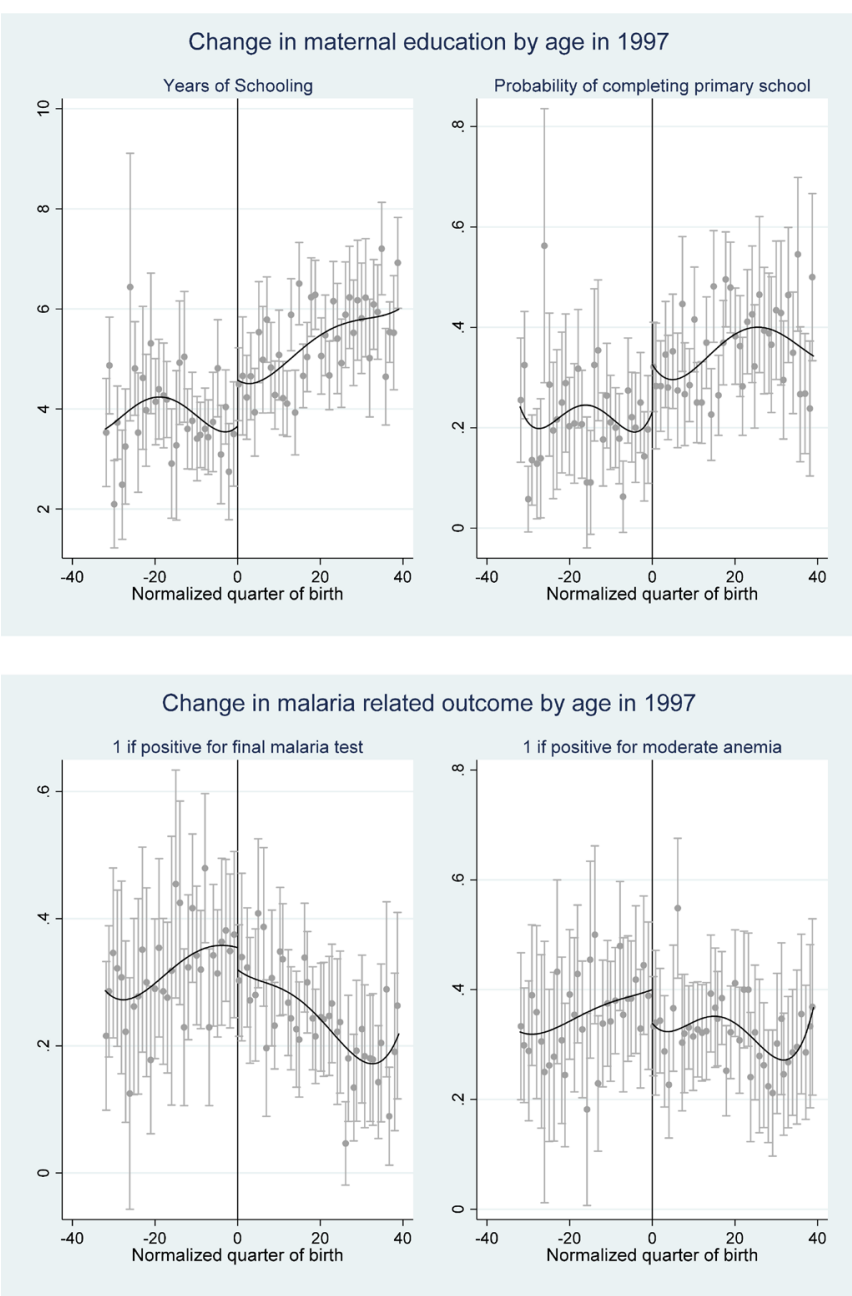

Figure 5 Changes in maternal education and child malaria by maternal birth cohort in Uganda.

cross-sectional, household surveys that included malaria biomarker collections for children younger than 5 years of age. ${ }^{12-14}$ In the 2012, 2014 and 2017 surveys, 3404,

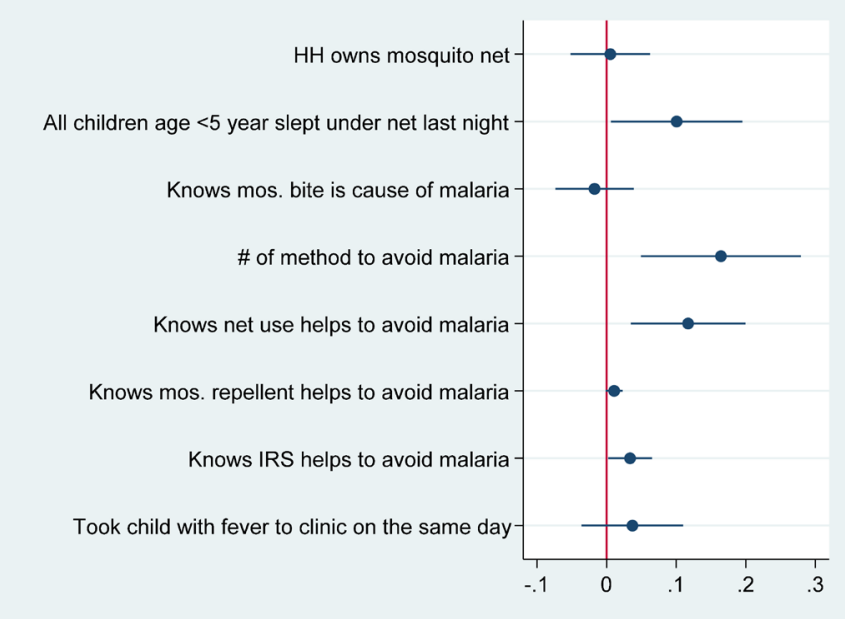

Figure 6 The effects of maternal schooling on the mechanism outcome. HH, household; IRS, indoor residual spraying.

3405 and 3729 households were interviewed, respectively. The response rates were $98 \%, 99 \%$ and $99.8 \%$, respectively. ${ }^{38-40}$ All girls/women aged $15-59$ years were eligible for individual interviews. In addition, I obtained data from the MMIS concerning testing for malaria in children aged 0-59 months using a finger-prick blood collection method. All children aged 0-59 months in the households selected for MMIS were eligible for biomarker collection, and $97 \%$ for 2012 and 2014 MMIS, and $99 \%$ for 2017 MMIS of eligible children were tested for malaria with microscopy in both years. ${ }^{38-40}$ Key information on birth year, age and mothers' educational attainment were available for $99 \%$ of the respondents.

I excluded children of mothers born in 1970 or earlier and those born in 1989 or later because those women were likely to be exposed to other education reform programmes. Children of mothers who were aged $<18$ years were also excluded because they may still have been

\begin{tabular}{|c|c|c|c|}
\hline & Exposure & Outcome & Effect estimate (SE) \\
\hline Model 1: 2SLS, slope change in year of birth & Years of schooling & Malaria positive & $-6.3^{\star \star}(2.6)$ \\
\hline Model 2: 2SLS, quadratic in year of birth & Years of schooling & Malaria positive & $-6.3^{\star \star}(2.9)$ \\
\hline Model 3: 2SLS, quadratic in maternal age & Years of schooling & Malaria positive & $-6.4^{*}(3.9)$ \\
\hline Model 4: 2SLS, control birth order & Years of schooling & Malaria positive & $-9.3^{*}(5.4)$ \\
\hline Model 5: 2SLS, narrow window & Years of schooling & Malaria positive & $-8.2^{*}(4.8)$ \\
\hline Model 6: 2SLS, exclude 1982 cohort & Years of schooling & Malaria positive & $-7.4^{* \star *}(3.4)$ \\
\hline Model 7: 2SLS, Malawi & Years of schooling & Malaria positive & $-7.1^{\star \star \star}(2.6)$ \\
\hline Model 8: ITT, education $=0$ & Reform exposure & Malaria positive & $-0.7(5.0)$ \\
\hline
\end{tabular}

Coefficient and SEs are multiplied by 100 and reported on a percentage point scale. Models 1-6 were estimated by IV-2SLS method, in which binary indicator for being born 1982 or later was used as an excluded IV for maternal years of schooling. Model 7 was estimated by IV-2SLS method, in which binary indicator for being born 1979 or later was used as an excluded IV for maternal years of schooling. Model 8 is estimated by ordinary least square method. The entire model controls for a full set of children's age-in-months indicators, linear term of maternal year of birth, indicator for survey year, indicator for survey month, indicator for child sex, indicator for ethnic groups and maternal age. No weights were used.

${ }^{*} \mathrm{P}<0.05,{ }^{* *} \mathrm{p}<0.01,{ }^{* * *} \mathrm{p}<0.001$

ITT, intention-to-treat ; IV, instrumental variable; 2SLS, two-stage least square. 
receiving formal education at the time of the survey. I also excluded women with no children aged $<5$ years because my primary interests were the effects of maternal education on childhood malaria control.

Primary exposure was defined as mothers' educational attainment (in years) at the time of the survey. The primary outcome was their children's malaria status at the time of the survey. I defined a binary variable that takes the value of unity if the respondent woman born in a cohort was exposed to the 1994 reform, otherwise 0 . Considering the share of individuals attending primary school in 1994 by age, those born in 1980 or later (ie, $\leq 13$ years as of 1 January 1994) were defined as exposed. I excluded the 1979 pivotal cohort from the main analysis. ${ }^{41}$ I also tested the sensitivity of the results by including the 1979 cohort for a robustness check. In the analysis, I controlled for maternal year of birth, maternal ethnicity, children's age in months, sex and month and year of the survey. Online supplementary appendix table S4 presents the results of the detailed replication in Malawi.

\section{DISCUSSION}

The present study revealed that length of maternal education reduces the probability that children will contract malaria in Uganda. Length of maternal education was positively associated with better use of ITNs. The results suggest an empirical association between maternal schooling and the children's risk of malaria infection previously reported in the existing observational studies potentially underestimated the true causal effects. The current results are interpreted as causal because exposure to the reform is considered random, and the reform is likely to change child malaria risk only through its effects on maternal schooling.

Theoretically, average treatment effects estimated by the IV-2SLS method are interpreted as 'local' treatment effects for a subset of the population. First, I exploited exogenous variation in women's educational attainment caused by the reform at the level of primary education. Therefore, the estimate is local to the population who are induced to stay longer at primary education. It may be surprising that even a low level of education (ie, primary) had modest protective effects on childhood malaria control, and the effects of higher education may be even stronger. Second, the present estimates are local to the population-so-called compliers-who attained more schooling if the reform was implemented when they were 14 years of age, but not in the absence of the reform. Online supplementary appendix table S5 uses exogenous characteristics of the mothers to describe the relative characteristics of the compliers compared with the overall sample by following the study by Marbach and Hangartner. ${ }^{42}$ The results show that the compliers are likely to live in Kampala (capital of Uganda) or an urban area and belong to a minority ethnic group. Third, the estimated effects are local to the context of malaria-endemic countries. Thus, the association between maternal education and childhood malaria control may differ in countries with a lower prevalence of malaria in children. Fourth, the findings are local to the context, in which ITNs; IRS and malaria treatment, such as artemisinin-based combination therapy, are plausibly available. When the survey was conducted in 2014, $90 \%$ of households owned at least one ITN and $45 \%$ of pregnant women received at least two doses of IPT. ${ }^{25} 26$ Therefore, it may not be plausible to generalise the findings of the earlier cohorts who were raised before malaria control interventions had not rolled out sufficiently in Uganda. However, given that these malaria control methods are becoming practically available in malariaendemic countries, the results should inform present and future malaria control strategies. Having consistent evidence from Malawi supports the high generalisability of the current findings.

The 2SLS estimates outweigh the OLS estimates for distinct reasons other than the LATE interpretation. One possible explanation is that unobserved confounding factors that positively affect length of maternal schooling are also positively correlated with children's malaria risk, or vice versa. For example, a mother from an urban area may attain more education because the distance to the nearest primary school is short; simultaneously, her child may be more likely to contract malaria, potentially because the household has less access to ITNs. ${ }^{25} 26$ In this scenario, estimate from naive OLS method is biassed towards 0 .

The findings of the present study also present new evidence regarding the reform that removed the tuition fees for primary school that has commonly been implemented in sub-Saharan African countries including Uganda and Malawi. Although these free primary education reforms have often been anecdotally criticised as chaotic due to school congestion and as having little impact on student learning, the evidence shows that the reform had beneficial impacts on female education and malaria eradication among children over the long term.

The current study had several limitations. First, participation rates for a child malaria blood test were not perfect, although they were very high $(97 \%)$. No discontinuity in participation rates were observed around the cut-off. Thus, the composition of mother whose child provided the malaria biomarker samples was comparable between the exposed and the unexposed cohort. Second, some children aged $<5$ years may contract malaria before their mother completes education. This is theoretically possible, but empirically very unlikely, because this study excluded women who were aged $<18$ years at the time of the survey, and few women attend primary schools after age 18 years. Third, as described in the 'Methods' section, some of the variables were measured based on the mothers' self-reported information; for this reason, theoretically, there is a potential for misclassifications, although this is empirically unlikely for several reasons. For example, one primary advantage of using 'year' of birth rather than 'year-month' of birth is that the type of 
information is less likely to be misclassified. Additionally, information on child age in months and ethnicity was unlikely to be misclassified because children's birthdays are recorded on child health cards, and ethnicity is objectively observable. Furthermore, if such a misclassification were to exist, it could be expected to occur randomly and be uncorrelated with the exposure to the reform; thus, the estimated effects of maternal length of education on children's malaria risk would not be biassed. Fourth, the reform may have reduced maternal mortality, and thus the composition of the observed sample may systematically differ between the exposed and the unexposed individuals. To test if this is the case, I compared the cohort size by birth cohort, and found no significant increase in the cohort size among the 1982 or later cohorts compared with 1981 or earlier cohorts (online supplementary figure S2). Thus, selective mortality of the mother is unlikely to bias my estimate. Fifth, the reform may reduce the child mortality. Thus, the composition of the observed sample of children may differ between the exposed cohort and the unexposed cohort. However, as reported in the existing study, the reform was shown to have no effects on the probability of infant mortality. Hence, selective child mortality is also unlikely to bias the estimates. Sixth, available data allowed me to observe children's probability of contracting malaria only up to 59 months of age. Although limited, this is a crucial period, when malaria often causes loss of life and neurocognitive impairment. ${ }^{43}$ Since the benefits of maternal education on malaria control are likely to be applicable to older children, and even to adults, the true cost-effectiveness of the intervention may be even greater than the current estimates. Seventh, although this is not a limitation, the reforms may have an impact on the prevalence of mothers without children. Online supplementary appendix table S6, however, shows that the exposure to the 1997 reform was not significantly associated with the probability that a woman had no children at the time of the survey. Lastly, because of the survey dates and inclusion criteria, the children of the first exposed 1982 cohort were born when the mothers were aged 21-31 years at the time of the survey. This is the period during which many women are pregnant in Uganda. Although teenage pregnancies are less common than pregnancies among women in their $20 \mathrm{~s},{ }^{25}{ }^{26}$ future research should examine the effects of maternal education on malaria incidence in these younger mothers.

Promoting access to primary education for women had a substantial effect on childhood malaria control in Uganda. The cost-effectiveness ratio per DALY averted of the primary schooling intervention was high. Hence, reform to expand access to primary education for girls should be an effective means to improve malaria control, and it should be combined with other malaria vector control interventions since maternal education increased the appropriate use of bednets for young children.
Acknowledgements The author gratefully acknowledges the financial support from JSPS (grant number 19K13677) and Hitotsubashi University. And Yutaro Takayasu for providing excellent research assistance.

Contributors KM contributed to the conception, design of the work, data analysis and interpretation, drafting the article, critical revision of the article and final approval of the text.

The authors have not declared a specific grant for this research from any funding agency in the public, commercial or not-for-profit sectors.

\section{Competing interests None declared.}

Patient consent for publication Not required.

Ethics approval The present study was reviewed by the Hitotsubashi University Institutional Review Board and considered exempt from full ethics review because the analysis was based on a publicly available, anonymised dataset. Nonetheless, this study conformed to the principles embodied in the Declaration of Helsinki.

Provenance and peer review Not commissioned; externally peer reviewed.

Data availability statement Data may be obtained from a third party and are not publicly available.

Open access This is an open access article distributed in accordance with the Creative Commons Attribution Non Commercial (CC BY-NC 4.0) license, which permits others to distribute, remix, adapt, build upon this work non-commercially, and license their derivative works on different terms, provided the original work is properly cited, appropriate credit is given, any changes made indicated, and the use is non-commercial. See: http://creativecommons.org/licenses/by-nc/4.0/.

\section{ORCID iD}

Kazuya Masuda http://orcid.org/0000-0003-3103-0424

\section{REFERENCES}

1 Vos T, Allen C, Arora M, et al. Global, regional, and national incidence, prevalence, and years lived with disability for 310 diseases and injuries, 1990-2015: a systematic analysis for the global burden of disease study 2015. Lancet 2016;388:1545-602

2 Tusting LS, Willey B, Lucas $\mathrm{H}$, et al. Socioeconomic development as an intervention against malaria: a systematic review and metaanalysis. Lancet 2013;382:963-72.

3 Safeukui-Noubissi I, Ranque S, Poudiougou B, et al. Risk factors for severe malaria in Bamako, Mali: a matched case-control study. Microbes Infect 2004;6:572-8.

4 Clouston SAP, Yukich J, Anglewicz P. Social inequalities in malaria knowledge, prevention and prevalence among children under 5 years old and women aged 15-49 in Madagascar. Malar $J$ 2015;14:499.

5 Rosenzweig M, Schultz T. The behavior of mothers as inputs to child health: the determinants of birth weight, gestation, and rate of fetal growth. In: Economic aspects of health. University of Chicago Press, 1982: 53-92.

6 Dike N, Onwujekwe O, Ojukwu J, et al. Influence of education and knowledge on perceptions and practices to control malaria in Southeast Nigeria. Soc Sci Med 2006;63:103-6.

7 Ahmed SM, Haque R, Haque U, et al. Knowledge on the transmission, prevention and treatment of malaria among two endemic populations of Bangladesh and their health-seeking behaviour. Malar J 2009;8:173.

8 Tang S, Ji L, Hu T, et al. Public awareness of malaria in the middle stage of national malaria elimination programme. A cross-sectional survey in rural areas of malaria-endemic counties, China. Malar $J$ 2016;15:373.

9 Masuda K, Yamauchi C. How does female education reduce adolescent pregnancy and improve child health? Evidence from Uganda's universal primary education for fully treated cohorts. J Dev Stud 2018:1-24.

10 Keats A. Women's schooling, fertility, and child health outcomes: evidence from Uganda's free primary education program. J Dev Econ 2018;135:142-59.

11 Njama D, Dorsey G, Guwatudde D, et al. Urban malaria: primary caregivers' knowledge, attitudes, practices and predictors of malaria incidence in a cohort of Ugandan children. Trop Med Int Health 2003;8:685-92.

12 Ouattara AF, Raso G, Edi CVA, et al. Malaria knowledge and longlasting insecticidal net use in rural communities of central Côte d'Ivoire. Malar J 2011;10:288. 
13 Noor A, Omumbo J, Amin A, et al. mother's education and physical access as determinants of retail sector net use in rural Kenya. Malar J 2006;5:5

14 Shah JA, Emina JBO, Eckert E, et al. Prompt access to effective malaria treatment among children under five in sub-Saharan Africa: a multi-country analysis of national household survey data. Malar $J$ 2015;14:329.

15 Shin-Yi C, Liu JT, Grossman M, et al. Parental education and child health: evidence from a natural experiment in Taiwan. Am Econ $J$ Appl Econ 2010;2:33-61.

16 Glewwe P. Why does mother's schooling raise child health in developing countries? Evidence from Morocco. J Hum Resour 1999;34:124-59.

17 Lindeboom M, Llena-Nozal A, van der Klaauw B. Parental education and child health: evidence from a schooling reform. J Health Econ 2009;28:109-31.

18 Deininger K. Does cost of schooling affect enrollment by the poor? universal primary education in Uganda. Econ Educ Rev 2003;22:291-305

19 UNESCO, 2019. Available: http://stats.uis.unesco.org/unesco [Accessed 1 Feb 2019].

20 Avenstrup R, Liang X, Kenya NS. Lesotho, Malawi and Uganda: universal primary education and poverty reduction. Africa regional educational publications. Washington, DC: World Bank, 2004

21 Ministry of Education and Sports, the Republic of Uganda. The Ugandan experience of universal primary education (UPE) 1999.

22 Nishimura M, Yamano T, Sasaoka Y. Impacts of the universal primary education policy on educational attainment and private costs in rural Uganda. Int J Educ Dev 2008;28:161-75.

23 Behrman JA. The effect of increased primary schooling on adult women's HIV status in Malawi and Uganda: universal primary education as a natural experiment. Soc Sci Med 2015;127:108-15.

24 Uganda Ministry of Health, National Malaria Control Division, Surveillance Monitoring \& Evaluation Unit. National malaria annual report 2017-2018. Kampala, Uganda, 2019.

25 Uganda Bureau of Statistics (UBOS) and ICF Macro. Uganda malaria indicator survey 2009. Calverton, Maryland, USA: UBOS and ICF Macro, 2010.

26 Uganda Bureau of Statistics (UBOS) and ICF International. Uganda malaria indicator survey 2014-15. Kampala, Uganda, and Rockville, Maryland, USA: UBOS and ICF International, 2015.

27 Angrist JD, Imbens GW, Rubin DB. Identification of causal effects using instrumental variables. J Am Stat Assoc 1996;91:444-55.

28 Imbens GW, Angrist JD. Identification and estimation of local average treatment effects. Econometrica 1994;62:467-75.
29 Lee DS, Lemieux T. Regression discontinuity designs in economics. $J$ Econ Lit 2010;48:281-355

30 De Neve J-W, Fink G, Subramanian SV, et al. Length of secondary schooling and risk of HIV infection in Botswana: evidence from a natural experiment. Lancet Glob Health 2015;3:e470-7.

31 UNESCO Institute for Statistics. Education indicators, 2019. Available: http://data.uis.unesco.org/index.aspx?queryid=189\# [Accessed 24 Feb 2019].

32 World Bank, 2019. Available: https://data.worldbank.org/indicator/ SP.DYN.TFRT.IN?locations=UG [Accessed 24 Feb 2019].

33 Gunda R, Chimbari MJ. Cost-Effectiveness analysis of malaria interventions using disability adjusted life years: a systematic review. Cost Eff Resour Alloc 2017:15:10.

34 White MT, Conteh L, Cibulskis R, et al. Costs and cost-effectiveness of malaria control interventions - a systematic review. Malar J 2011;10:337.

35 Goodman CA, Coleman PG, Mills AJ. Cost-Effectiveness of malaria control in sub-Saharan Africa. The Lancet 1999;354:378-85.

36 Penny MA, Verity R, Bever CA, et al. Public health impact and cost-effectiveness of the RTS,S/AS01 malaria vaccine: a systematic comparison of predictions from four mathematical models. The Lancet 2016;387:367-75.

37 World health statistics. 2018: monitoring health for the SDGs, sustainable development goals. Geneva: World Health Organization, 2018.

38 National Malaria Control Programme and ICF. Malawi malaria indicator survey 2017. Lilongwe, Malawi, and Rockville, Maryland, USA: NMCP and ICF international, 2018

39 National Malaria Control Programme (NMCP) [Malawi] and ICF International. Malawi malaria indicator survey (MIS) 2014. Lilongwe, Malawi, and Rockville, Maryland, USA: NMCP and ICF International, 2014.

40 National Malaria Control Programme [Malawi] and ICF International. Malawi malaria indicator survey (mis) 2012. Lilongwe, Malawi, and Calverton, Maryland, USA: NMCP and ICF International, 2012.

41 Makate M, Makate $\mathrm{C}$. The causal effect of increased primary schooling on child mortality in Malawi: universal primary education as a natural experiment. Soc Sci Med 2016;168:72-83.

42 Marbach M, Hangartner D. Profiling compliers and non-compliers for instrumental variable analysis. SSRN Journal 2019.

43 Bangirana $\mathrm{P}$, Opoka RO, Boivin MJ, et al. Severe malarial anemia is associated with long-term neurocognitive impairment. Clin Infect Dis 2014;59:336-44. 